\title{
Life After Eruption: Best of 2009-2013
}

\author{
C. Tappert ${ }^{1}$, A. Ederoclite ${ }^{2}$, L. Schmidtobreick ${ }^{3}$, N. Vogt $^{1}$ \\ ${ }^{1}$ Departamento de Física y Astronomía, Universidad de Valparaíso, Chile \\ ${ }^{2}$ Centro de Estudios de Física del Cosmos de Aragón, Teruel, Spain \\ ${ }^{3}$ European Southern Observatory, Santiago, Chile \\ Corresponding author: claus.tappert@uv.cl
}

\begin{abstract}
From our ongoing survey to study the post-nova population we present details on the four objects V728 Sco, AR Cir, V972 Oph and X Cir.
\end{abstract}

Keywords: cataclysmic variables - novae - individual: V728 Sco - AR Cir - V972 Oph - X Cir.

\section{Introduction}

In 2009 we have begun a survey on post-novae that had erupted before 1980, in order to address the lack of identified post-nova systems. Scope, methods and the current state of the survey are presented by Ederoclite et al. elsewhere in this volume. In the present article we take a closer look at four objects from our survey with peculiar properties that deserve further investigations. A detailed analysis of three of these systems, V728 Sco, AR Cir and V972 Oph, can be found in Tappert et al. (2013a, b), and we here only briefly summarize the results.

\section{V728 Sco}

The CV corresponding to Nova Sco 1862 was recovered as a $\mathrm{V}=18.5 \mathrm{mag}$ object roughly 2 arcmin north-west of its suspected position (Tappert et al. 2012). Its spectrum presents - for an old nova - unusually strong emission lines. Photometric time-series revealed the object to be eclipsing with an orbital period $P_{\text {orb }}=3.32 \mathrm{~h}$. This places V728 Sco within the regime of the SW Sex type systems, CVs that are characterized by very high mass-transfer rates $\dot{M}$ (e.g., Rodríguez-Gil et al. 2007a, b; see also Schmidtobreick \& Tappert, this volume). Furthermore, old novae in general are expected to run high $\dot{M}$ due to irradiation of the secondary star by the eruption-heated white dwarf (Kovetz et al. 1988), and in general this is confirmed by observations (Iben et al. 1992). For example, the old nova RR Pic, with $P_{\text {orb }}=3.48 \mathrm{~h}$, does indeed share the characteristics of an SW Sex star (Schmidtobreick et al. 2003a, 2008). V728 Sco, on the other hand, appears to have a lower $\dot{M}$ than expected. Evidence for this does not only stem from the spectroscopic appearance, but also from the long-term behaviour that shows the system to vary between a low and a $\sim 1.5$ mag brighter state. While the data coverage is still very sparse, it looks like this brighter state is in general short-lived and bears resemblance to the outbursts of dwarf novae.

Analysis of one eclipse that was observed in the low state provided an estimate of the radius of the eclipsed source to $R=0.09 R_{\odot}$, which is significantly larger than any feasible solution for a white dwarf. We interpret this result as the presence of a hot inner disc, such as has been invoked by Schreiber et al. (2000) to explain the low-amplitude high-frequency outbursts that are observed in some post-novae and nova-like stars (e.g., Honeycutt et al. 1998), and would also fit the longterm behaviour of V728 Sco. Our eclipse data provide the first direct evidence for the presence of such disc.

\section{AR Cir}

AR Cir was a comparatively slow nova that erupted in 1906. It was recovered by Duerbeck \& Grebel (1993). Time-series spectroscopy taken by us on three consecutive nights in 2012 yielded $P_{\text {orb }}=5.14 \mathrm{~h}$. The spectrum shows a moderately strong $\mathrm{H} \alpha$ emission line on a red continuum, the latter being very probably entirely caused by interstellar reddening rather than due to the contribution of the secondary star. The R-band brightness shows a clear modulation with $P_{\text {orb }}$ and an amplitude of $\sim 0.3 \mathrm{mag}$, indicating that the inclination is comparatively high. The $\mathrm{H} \alpha$ line profile shows an intriguing variability in that at certain orbital phases, and strongest at phase 0.5 (corresponding to the maximum of the R-band light curve), a P Cyg component becomes visible in the form of a high-velocity blue absorption and a red emission component. We interpret 
this as an optically thick outflow and determine a rough estimate of the projected outflow velocity to $700 \pm 100$ $\mathrm{km} / \mathrm{s}$.

\section{$4 \quad$ V972 Oph}

Time-series spectroscopic observations of this post-nova (Nova Oph 1957) yield an orbital period $P_{\text {orb }}=6.75 \mathrm{~h}$ thus making it a member of the minority of novae with comparatively long orbital periods (see Ederoclite et al., this volume). Examination of the profile of the $\mathrm{H} \alpha$ emission line reveals an additional emission at $\sim 6580$ $\AA$. This component moves roughly parallel to the main $\mathrm{H} \alpha$ component. This practically excludes scenarios of an additional $\mathrm{H} \alpha$ component from the secondary star or from the hot spot, as well as a nebular line like [NII] $\lambda 6584$. The absence of an absorption line blueward of $\mathrm{H} \alpha$ lets an origin in an outflow, such as assumed for AR Cir, also appear unlikely. Instead we tentatively identify that line with the CII $\lambda \lambda 6578 / 6583$ doublet. To our knowledge, this is the first time that this line is observed in a CV in this form (it had also be identified as originating in the outflowing material of the helium nova V445 Pup; Iijima \& Nakanashi 2008).

While carbon emission has been observed before in some post-novae (e.g., Schmidtobreick et al. 2003b, Bianchini et al. 2012), this concerns mostly the blue part of the spectrum. Unfortunately the available spectra of V972 Oph by Zwitter \& Munari (1996) and Ringwald et al. (1996) that cover that range have too low $\mathrm{S} / \mathrm{N}$ to confirm the presence of such lines in V972 Oph.

The weakness of the $\mathrm{H} \alpha$ emission line (which has an equivalent width of $2 \AA$ ) certainly helped in the detection of the CII line. This raises the question whether this line is not more frequently present in CVs, but hidden away in the wings of a stronger $\mathrm{H} \alpha$ emission. A quick examination of seven novae finds three more systems with likely excess emission at $\sim 6580 \AA$. As a caveat we note that this list includes AR Cir, where we find an outflow as a more likely explanation. However, the other systems lack an accompanying blue emission or absorption component that would suggest such phenomenon as the origin.

\section{$5 \quad \mathrm{X}$ Cir}

The eruption of Nova Cir 1927 was reported by Becker (1929). The aftermath of the nova is little studied. Duerbeck (1987) classifies it as a slow nova with $t_{3}=$ 170 d. Woudt \& Warner (2002) identified a possible candidate for the post-nova due to short-term variability, but a spectrum taken by Mason \& Howell (2003) revealed that object to have a reddish continuum without emission lines.

Our UBVR photometry from May 2009 (Fig. 1, top) allows for a number of candidates based on colour and distance from the reported coordinates. In 2012 we obtained spectroscopic data for five of them with the MOS mode of FORS2 at the ESO-VLT (UT1). The post-nova was confirmed to be an object roughly 1.2 arcmin north-west of its suspected position. The spectrum shows comparatively strong emission lines (equivalent width of $\mathrm{H} \alpha \sim 30 \AA$ ) of the Balmer and HeI series (Fig. 1, bottom).
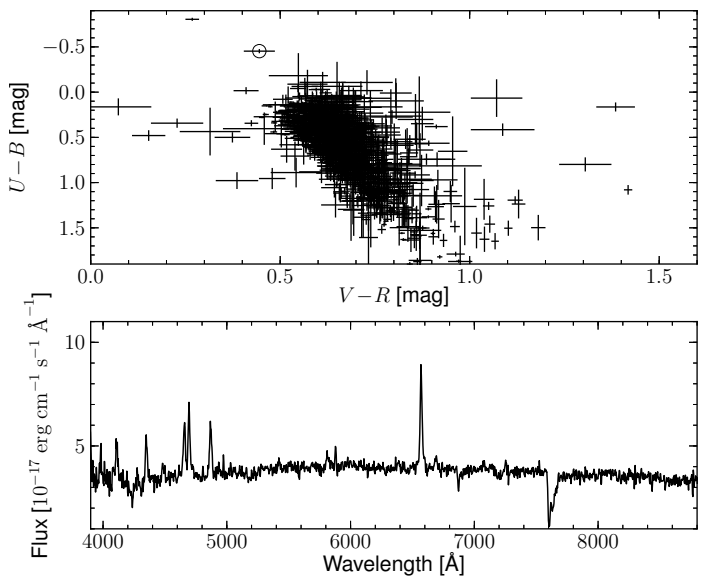

Figure 1: Top: Colour-colour diagram of the field $(4.5 \times 4.5 \mathrm{arcmin})$ of $\mathrm{X}$ Cir. The confirmed post-nova is marked by a circle. Bottom: Low-resolution spectrum of the post-nova.

While being situated in a promising place in the colour-colour diagram, the later confirmed post-nova was not our best candidate, but in fact rather represented the very bottom of our list. The reason for this is that our photometric data showed a larger point-spread function (PSF) than for the other stars in the field, indicating an extended object. This was later confirmed by the 2D spectroscopic data. An examination of the latter showed that the emission lines appear to be confined to the one part of the spectroscopic PSF that corresponds to the eastern part of the photometric PSF. Our preliminary conclusion is therefore that the extension is not due to a shell, but that this is an unresolved $(<0.5$ arcsec) visual binary.

The spectrum of $\mathrm{X}$ Cir presents a prominent HeII 4686 line, and even, albeit much weaker, HeII 5411 (Fig. 2). Such strong presence of HeII is often a signature of a magnetic system. On the other hand, the HeI lines are distinctively double-peaked with a deep central valley, indicating the presence of an accretion disc. A possible explanation is that $\mathrm{X}$ Cir is an intermediate polar. The HeII 5411 line is also a transient feature. In our three spectra, taken with a time separation of $\sim 3 \mathrm{~h}$ between the first and the second, and $\sim 24$ $\mathrm{h}$ between the second and the third, the line is strongest in the first (which is the one in Fig. 2), not detected in 
the second, and weakly present in the third spectrum (Fig. 1). Since the individual integration times amount to $15 \mathrm{~min}$, this could be an orbital effect. The HeII lines and the Balmer lines are single-peaked, suggesting that at least a major part of their profile has an origin different from that of HeI.

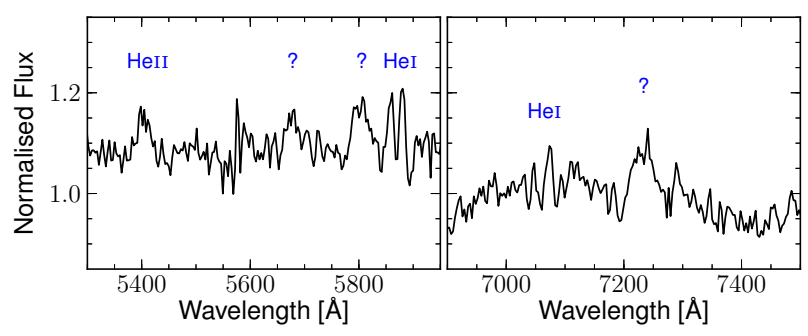

Figure 2: Close-up on the spectrum of X Cir. The label mark the line identifications; a question mark is used for the three unidentified lines. Note the deep central valleys in the double-peaked profile of the two present HeI lines.

We furthermore find three emission lines that we have not yet been able to identify (Fig. 2). Taking into account the observed shift of the identified lines, we estimate their corresponding rest wavelengths to $\geq 5678$, $\geq 5805, \geq 7233$, respectively. Examining lists of spectral lines (e.g., Coluzzi 1999) does not present obvious suspects for an identification. We note that the profiles are also single-peaked, so that these probably do not originate exclusively in the accretion disc.

We derive the 'quiescent' brightness of X Cir in the $V$ filter to $19.3 \mathrm{mag}$. The reported photographic value of the eruption maximum is 6.5 mag (Duerbeck 1987). Assuming that the effect of the difference between the filters is $<0.5 \mathrm{mag}$, this yields an eruption amplitude $>12$ mag. This places it a bit off the fit to the rateof-decline / eruption-amplitude relation (Ederoclite et al., this volume; note that X Cir is not yet included in their figure), but still within the larger scatter.

\section{Summary}

We have presented four post-novae with apparently peculiar properties. V728 Sco is one of the few objects that defies the general trend that post-novae are CVs with very high mass-transfer rates $(\dot{M})$. Finding such objects will be very important to answer the question whether post-novae in general have high $\dot{M}$ as a consequence of the nova eruption, or if high $\dot{M} \mathrm{CV}$ s are just more likely to erupt as a nova. V728 Sco is furthermore deeply eclipsing, which presents an excellent and rarely found opportunity to determine accurate system parameters of a nova.

AR Cir, on the other hand, while with more than 100 yr having passed since its eruption can already be counted among the 'old' old novae, apparently still drives such high $\dot{M}$ that it causes an outflow of material that is visible in the optical spectrum. Since our data only covers the spectral range next to $\mathrm{H} \alpha$ it would be interesting to examine other spectral ranges and lines for this phenomenon to gain more information on the outflow.

In V972 Oph we detect an unusual emission line that we attribute to carbon. We argue that such line could in principle be more frequent in CVs because it can be easily hidden in the wings of the usually strong $\mathrm{H} \alpha$ emission that is exceptionally weak in V972 Oph. The currently available data on the blue spectral range has too low $\mathrm{S} / \mathrm{N}$ to examine it for other tracers of the presence of carbon in V972 Oph, thus a higher quality spectrum of that region would be most welcome. The carbon line in V972 Oph moves parallel to $\mathrm{H} \alpha$ and thus likely originates in the accretion disk or the bright spot, or on the secondary star itself. In any case it represents material from the secondary. It was either accreted from the expelled material during the nova eruption or produced in the secondary, which then could not have formed as a late-type main-sequence star, but must have undergone some nuclear evolution prior to its semi-detached CV phase. Thus, the study of carbon in (not only) post-novae bears relevance for our understanding on $\mathrm{CV}$ evolution.

The results on X Cir are still somewhat preliminary. The strong Balmer lines argue for a comparatively low $\dot{M}$, the prominent presence of HeII could point to a magnetic white dwarf, but at least is evidence for a hot region somewhere in the system, while the double-peaked HeI profiles indicate the presence of an accretion disc. A number of emission features yet remain to be identified which means that these lines are at least very rarely observed in CVs. Finally, the spectral appearance, the transient nature of HeII 5411 and the double-peaked HeI lines suggest a high inclination. There is therefore a good chance that the orbital period will be accessible comparatively easily via time-series photometry.

We hope that this presentation motivates further studies of those systems. While their properties may appear peculiar, they might not be exclusive to those objects. Because the number of detailed studies on post-novae is still fairly small, we cannot yet be certain if they are more representative of the rule or the exception.

\section{Acknowledgement}

This research was supported by FONDECYT Regular grant 1120338 (CT and NV). AE acknowledges support by the Spanish Plan Nacional de Astrononomía y Astrofísica under grant AYA2011-29517-C03-01. 


\section{References}

[1] Becker, F.: 1929, Astron. Nachr., 237, 71.

[2] Bianchini, A., Saygac, T., Orio, M., et al.: 2012, A\&A, 539, A94.

[3] Coluzzi, R.: 1999, VizieR Online Data Catalog, 6071.

[4] Duerbeck, H.W.: 1987, Space Sci. Rev., 45, 1. doi:10.1007/BF00187826

[5] Duerbeck, H.W., Grebel, E.K.: 1993, MNRAS, 265, L9. doi:10.1093/mnras/265.1.L9

[6] Honeycutt, R.K., Robertson, J.W., Turner, G.W.: 1998, AJ, 115, 2527.

[7] Iben, Jr., I., Fujimoto, M.Y., MacDonald, J.: 1992, ApJ, 384, 580. doi:10.1086/170900

[8] Iijima, T., Nakanashi, H.: 2008, A\&A, 482, 865.

[9] Kovetz, A., Prialnik, D., Shara, M.M.: 1988, ApJ, 325,828 .

[10] Mason, E., Howell, S.B.: 2003, A\&A, 403, 699.

[11] Ringwald, F.A., Naylor, T., Mukai, K.: 1996, MNRAS, 281, 192.

[12] Rodríguez-Gil, P., Schmidtobreick, L., Gänsicke, B.T.: 2007a, MNRAS, 374, 1359. doi:10.1111/j.1365-2966.2006.11245.x

[13] Rodríguez-Gil, P., Gänsicke, B.T., Hagen, H.-J., et al.: 2007b, MNRAS, 377, 1747. doi:10.1111/j.1365-2966.2007.11743.x

[14] Schmidtobreick, L., Tappert, C., Saviane, I.: 2003a, MNRAS, 342, 145. doi:10.1046/j.1365-8711.2003.06523.x

[15] Schmidtobreick, L., Tappert, C., Bianchini, A., Mennickent, R.E.: 2003b, A\&A, 410, 943.
[16] Schmidtobreick, L., Papadaki, C., Tappert, C., Ederoclite, A.: 2008, MNRAS, 389, 1345. doi:10.1111/j.1365-2966.2008.13641.x

[17] Schreiber, M.R. and Gänsicke, B.T. and Cannizzo, J.K.: 2000, A\&A, 362, 268

[18] Tappert, C., Ederoclite, A., Mennickent, R.E., et al.: 2012, MNRAS, 423, 2476.

[19] Tappert, C., Vogt, N., Schmidtobreick, L., et al.: 2013a, MNRAS, 431, 92.

[20] Tappert, C., Schmidtobreick, L., Vogt. N., Ederoclite, A.: 2013b, MNRAS, in press (arXiv 1302.5570).

[21] Woudt, P.A., Warner, B.: 2002, in: Classical Nova Explosions, Hernanz, M. \& José, J., eds., AIP Conf. Proc., Vol. 637, p.532.

[22] Zwitter, T., Munari, U.: 1996, A\&AS, 117, 449.

\section{DISCUSSION}

CHRISTIAN KNIGGE: A comment on V728 Sco: I think this is totally consistent with being a "normal" SW Sex star. It has the same V-shaped eclipse in the high state (and may be self-occulting), and several SW Sex stars, like DW UMa, also show high-state / lowstate behaviour.

CLAUS TAPPERT: It is true that the high-state eclipse very much resembles that of SW Sex stars. But I would argue that the other properties do not. We do not see any high-velocity wings (this might arguably be due to the quality of the spectra), and the emission lines are distinctively double-peaked. We need more long-term data to see if the system spends more time in low or high state, but so far my best bet would be that V728 Sco is in a somewhat transitory phase, not quite SW Sex, not quite dwarf nova. 